\title{
Hot Adsorbate-Induced Retardation of the Internal Thermalization of Nonequilibrium Electrons in Adsorbate-Covered Metal Nanoparticles
}

\author{
Christophe Bauer,* Jean-Pierre Abid, and Hubert H. Girault \\ Laboratoire d'Electrochimie Physique et Analytique, Faculté des Sciences de Base, Ecole Polytechnique \\ Fédérale de Lausanne, CH-1015 Lausanne, Switzerland
}

Received: January 10, 2006; In Final Form: February 7, 2006

\begin{abstract}
Femtosecond transient absorption spectroscopy has been used to investigate the electron-electron scattering dynamics in sulfate-covered gold nanoparticles of 2.5 and $9.2 \mathrm{~nm}$ in diameter. We observe an unexpected retardation of the absolute internal thermalization time compared to bulk gold, which is attributed to a negative feedback by the vibrationally excited sulfate molecules. These hot adsorbates, acting as a transient energy reservoir, result from the back and forth inelastic scattering of metal nonequilibrium electrons into the $\pi^{*}$ orbital of the sulfate. The vibrationally excited adsorbates temporarily govern the dynamical behavior of nonequilibrium electrons in the metal by re-emitting hot electrons. In other terms, metal electrons reabsorb the energy deposited in the hot sulfates by a mechanism involving the charge resonance between the sulfate molecules and the gold NPs. The higher surface-to-volume ratio of sulfate-covered gold nanoparticles of 2.5 $\mathrm{nm}$ leads to a stronger inhibition of the internal thermalization. Interestingly, we also note an analogy between the mechanism described here for the slow-down of electron-electron scattering in metal nanoparticles by the hot adsorbates and the hot phonon-induced retardation of hot charge carriers cooling in semiconductors.
\end{abstract}

Hybrid nanostructured materials can be seen as complex systems, which lie at the border of solid-state physics and supramolecular chemistry. Metal nanoparticles (NPs) protected with functional molecules can give a unique opportunity to investigate the coupling between electron transport dynamics and molecular structural changes beyond the Born-Oppenheimer approximation.

As the size decreases to the nanoscale range (shorter than the electron mean free path), the electronic properties of the metal can fall under the influence of surface molecular species. For example, evidence appeared that adsorbates can modify the electronic properties of small metal nanoparticles ${ }^{1-4}$ (below 20 $\mathrm{nm}$ ) by decreasing the surface plasmon lifetimes. This additional damping channel, which leads to a homogeneous broadening of the surface plasmon band, is called chemical interface damping, i.e., metal electrons tunnel back and forth into empty electronic states of adsorbates. ${ }^{5-7}$ The electronic structure of the gold/sulfate interface consists of a $\sigma$ donation and a $\sigma^{*}$ and $\pi^{*}$ back-donation mechanism: The occupied $\sigma$ orbitals of the sulfate give charge to the metal, while the unoccupied $\sigma^{*}$ and $\pi^{*}$ orbitals accept charge from the metal. ${ }^{8} \mathrm{~A}$ key characteristic of the present system is the coupling between gold electrons and sulfate vibrations via the partial occupation of the sulfate $\pi^{*}$ orbital by the gold electrons. This charge resonance leads to a strong chemical interface damping of the surface plasmon as revealed by the nonclassical broadening of the surface plasmon band. ${ }^{9}$ Thus, under equilibrium conditions, sulfate

* Corresponding author. Christophe Bauer, Laboratoire d'Electrochimie Physique et Analytique, Faculté des Sciences de Base, Ecole Polytechnique Fédérale de Lausanne, 1015 CH-Lausanne, Tél. + 412169331 56, Fax +412169336 67, E-mail address: christophe.bauer@epfl.ch. molecules affect the electronic properties of the gold nanoparticles by opening an additional damping channel for the surface plasmon. The next step would consist of investigating the effect of the adsorbates on the electron dynamics in a metal under conditions far from equilibrium. Such a situation can be achieved by the generation of nonequilibrium electrons in the sulfatecovered gold NPs upon the absorption of a femtosecond laser pulse.

The internal thermalization of nonequilibrium electrons, which corresponds to the establishment of an electronic temperature by electron-electron scattering, represents a typical many-body problem in condensed matter ${ }^{10}$ and controls the efficiency of many optoelectronic devices and interfacial processes. For example, inelastic nonequilibrium electrons tunneling at metal/ molecule interfaces plays a crucial role in catalysis ${ }^{11}$ and molecular electronics. ${ }^{12,13}$ More specifically, the dynamical behavior of nonequilibrium electrons (NEs) governs the yield of hot electron-mediated surface reactions on metal surfaces. ${ }^{14-16}$ The hot electron cooling dynamics ${ }^{17}$ as well as the mechanism of breathing modes excitation ${ }^{18}$ has already been reported for gold NPs. At present, the internal thermalization dynamics has been mainly investigated in bulk metals ${ }^{19-21}$ and naked metal nanoparticles. ${ }^{22,23}$ However, until now, information on electronelectron scattering dynamics in adsorbate-covered metal nanoparticles cannot be found in the literature.

Here, we wish to address the following question: How can adsorbates affect the dynamical behavior of NEs in a metal? For this purpose, we approach this problem for the first time with adsorbate/metal nanosystems (AMNs) by femtosecond transient absorption spectroscopy. Our approach relies on a recently developed method to investigate internal thermalization 

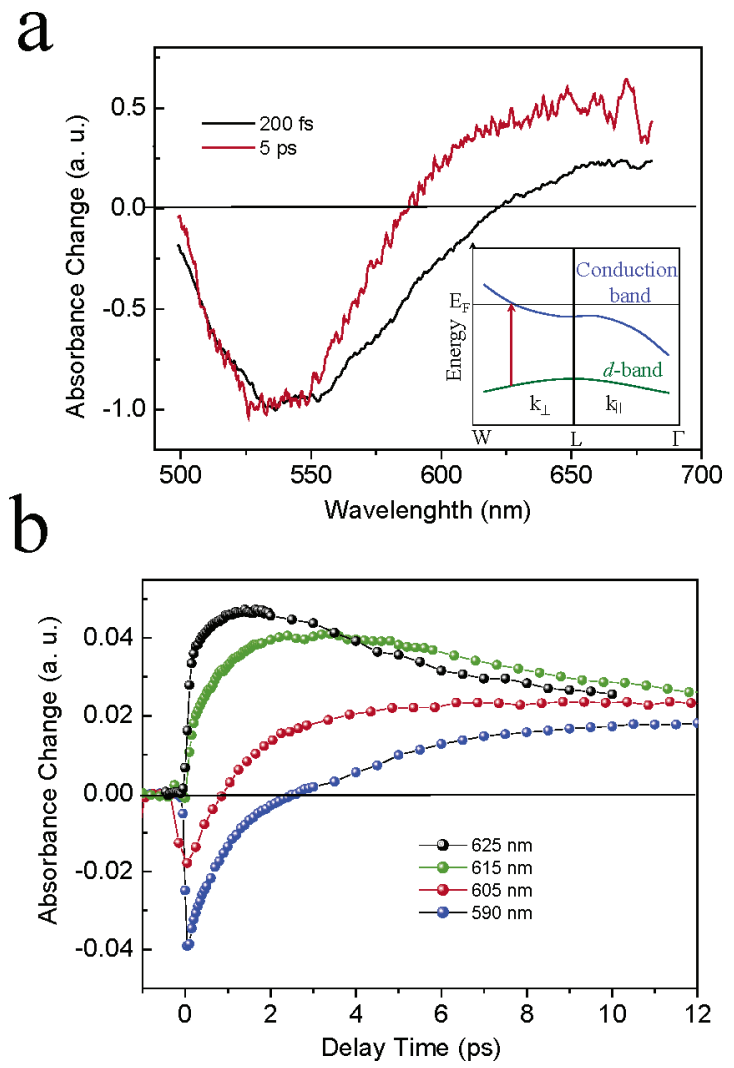

Figure 1. (a) Normalized differential transient absorption spectra of adsorbate/metal nanosystems of $2.5 \mathrm{~nm}$. The spectra were recorded after pumping at $480 \mathrm{~nm}$ with a pump fluence of $400 \mathrm{~nJ} /$ pulse. Inset Figure 1a: Schematic band structure of gold around the $L$-point in the Brillouin zone (after refs 20, 27, 28). The colored arrow illustrates the electronic transitions probed by femtosecond transient absorption spectroscopy. (b) Transient absorption data obtained for AMNs of $9.2 \mathrm{~nm}$ at probe wavelengths of $590,605,615$, and $625 \mathrm{~nm}$ with pump fluences of 450 , 420,425 , and $450 \mathrm{~nJ} / \mathrm{pulse}$, respectively.

by femtosecond transient absorption spectroscopy in bulk metals $^{20,21}$ and naked metal nanoparticles. ${ }^{22,23}$ It gives us the opportunity to directly compare the internal thermalization times between naked and adsorbate-covered metal NPs. The temporal evolution of the electron distribution around the Fermi level in the gold NPs is monitored by probing the absorption changes close to the threshold of the d-band to Fermi surface transitions in the vicinity of the $L$-point of the Brillouin zone $e^{20}$ (see inset of Figure 1a).

We use "soft" surface science to prepare the samples (see Supporting Information). ${ }^{24}$ The AMNs (sulfate-covered gold NPs) were grown in the pores of a metal oxide thin film by a heat treatment technique. ${ }^{25,9}$ Elementary Au NPs with an average diameter of $1.7 \pm 0.4 \mathrm{~nm}$ derivatized with mercapto succinic acid were first inserted by wet-grafting into the mesoporous thin film. This preparation method leads to sulfate-covered gold NPs as confirmed by our FT-IR and XPS spectroscopic data and as reported. ${ }^{25}$ Transmission electron microscopy revealed that the sizes of the resulting nanoparticles are $2.5 \pm 0.5 \mathrm{~nm}$ and $9.2 \pm 1.8 \mathrm{~nm}$. The femtosecond transient absorption setup features a compact CPA-2001 Ti:sapphire amplified femtosecond laser with a repetition rate of $1 \mathrm{kHz}$, a fundamental wavelength at $775 \mathrm{~nm}$, a pulse width of $120 \mathrm{fs}$, and an energy of $1 \mathrm{~mJ}$ per pulse. The output beam was split into three parts for pumping two double-stage noncollinear optical parametric amplifiers (NOPA) and to produce a white light continuum in a 2-mm-thick sapphire plate. For the monochromatic probing, a silicon diode was placed directly after the samples to measure the transmitted light intensity. The signal from the detector was connected to a lock-in amplifier tuned at $220 \mathrm{~Hz}$ by a chopper modulating the pump beam. ${ }^{9}$

Figures 1a shows differential transient absorption spectra recorded with delay times of $200 \mathrm{fs}$ and 5 ps upon pumping at $480 \mathrm{~nm}$ for $2.5 \mathrm{~nm}$ adsorbate-covered metal NPs and illustrates the different optical responses between the nonthermal and thermal electron distribution. The spectral signature of a nonthermal electron distribution consists of a broad featureless bleach with a minimum close to the interband transition threshold ( $\sim 520 \mathrm{~nm}) .{ }^{20,21}$ For a thermal distribution, the bleach is narrower, and the spectrum exhibits absorption for wavelengths longer than $580 \mathrm{~nm}$ as shown by the spectrum at $5 \mathrm{ps}$. The temporal evolution of the transient spectra shape, revealed by the shift of the intersection with the baseline from 620 to $580 \mathrm{~nm}$, reflects the transition from a nonthermal to a thermal distribution..$^{20,9}$ Figure $1 \mathrm{~b}$ shows the changes in signal shapes as a function of the probe wavelengths in the interesting spectral region where the signal becomes positive. The change of sign observed at 590 and $605 \mathrm{~nm}$ is direct evidence for the existence of a long-lived nonthermal distribution. ${ }^{20}$ By probing further from the d-band to Fermi surface transitions (with wavelengths longer than $520 \mathrm{~nm}$ ), more energetic electrons are monitored and faster dynamics (rise at 615 and $625 \mathrm{~nm}$ and recovery at 590 and $605 \mathrm{~nm}$ ) are observed in accordance with the Fermi liquid theory. ${ }^{26}$ The probe wavelength dependence is due to the curvatures of the d-band in the vicinity of the $L$-point of the Brillouin zone. ${ }^{20,27,28}$

Figure 2 shows transient absorption data obtained for 9.2 and $2.5 \mathrm{~nm}$ AMNs with different probe wavelengths and pump fluences. The bleach observed for a probe at $590 \mathrm{~nm}$ indicates the instantaneous generation of nonthermal electrons. The decay of nonthermal electrons can be followed by the recovery of the signal at $590 \mathrm{~nm}$. However, the signal also contains a weak contribution of electron-phonon interaction (see Supporting Information). The internal thermalization dynamics can unambiguously be followed by the rise of the signal recorded at 615 and $625 \mathrm{~nm}$, which reflect the establishment of an electronic temperature (build-up of a Fermi-Dirac distribution). The rises are very close to the instrument response at low pump fluence ,but a slow component appears by increasing the pump fluence (see Figure 2b,c). For high pump fluences, the maximum of the signal is reached only after delay times of $\sim 2.5$ and $\sim 1.5$ ps at 615 and $625 \mathrm{~nm}$, respectively. For the three probe wavelengths, the dynamics exhibit a common feature: the presence of two distinguishable dynamical regimes. Within regime A, the recoveries (Figure 2a) and the rises (Figure 2b,c) of the signals are pump fluence dependent and exhibit slower dynamics as the pump fluence increases, whereas in regime B, time constants become pump fluence independent. Additional measurements were performed with AMNs of $2.5 \mathrm{~nm}$ with $\sim 500$ gold atoms in total $(\sim 250$ atoms forms the core, whereas $\sim 250$ gold atoms are on the surface). Figure $2 \mathrm{~d}$ shows transient absorption data obtained at $625 \mathrm{~nm}$ for the $2.5 \mathrm{~nm}$ AMNs. In contrast to the $9.2 \mathrm{~nm}$ AMNs, no fast component is observed; however, we also note the presence of the two regimes $\mathrm{A}$ and B like for $9.2 \mathrm{~nm}$ AMNs.

To gain quantitative information on the internal thermalization, the signals have been fitted to extract the internal thermalization times (for details, see Supporting Information). For data obtained at $590 \mathrm{~nm}$, time constants increase from 190 to $1.26 \mathrm{ps}$ for 110 to $485 \mathrm{~nJ} /$ pulse, respectively, as shown in Figure 3a. Since the rates presented here at low pump fluence are too fast (190 fs), the recovery at $590 \mathrm{~nm}$ cannot only be 
a
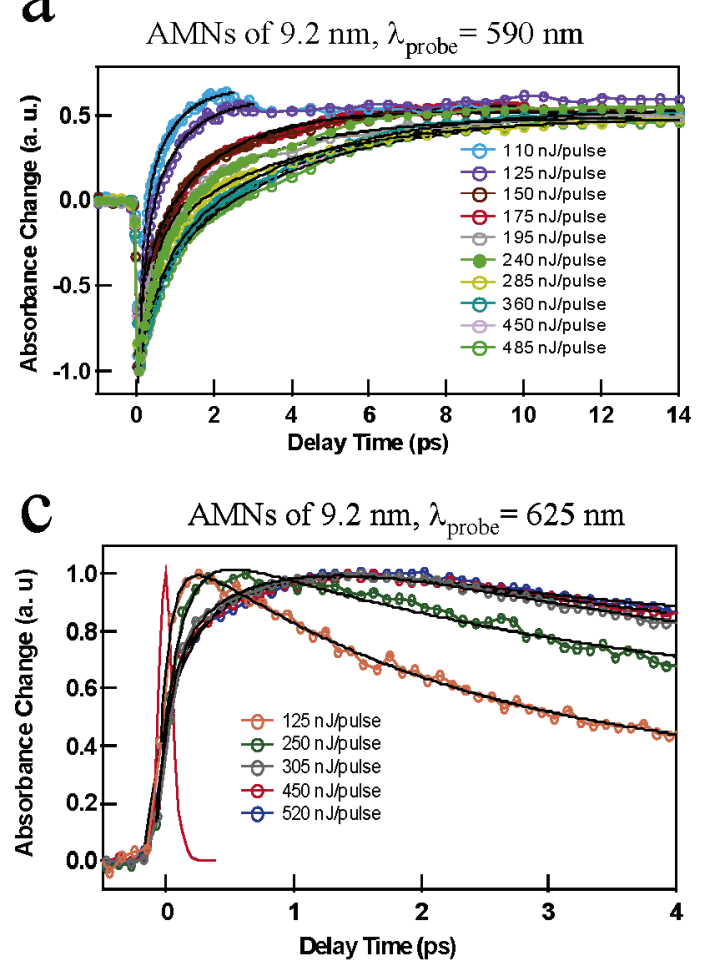

b

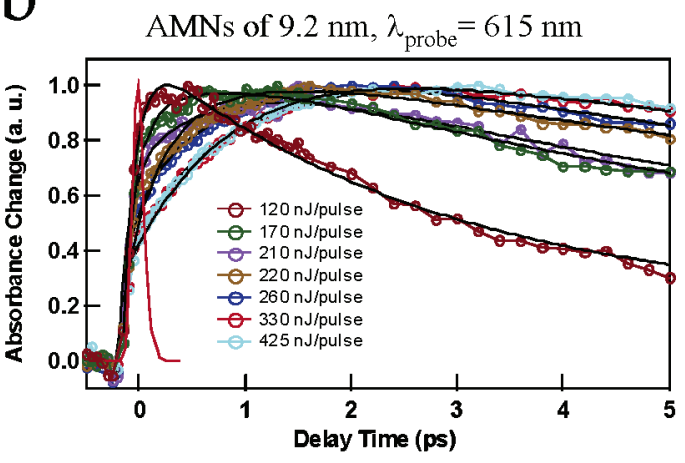

$\mathrm{d}$

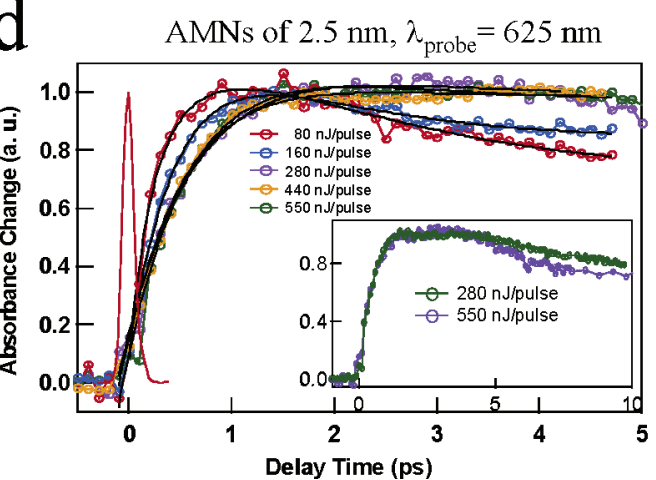

Figure 2. Normalized transient absorption data recorded at different probe wavelengths after pumping at $535 \mathrm{~nm}$ with different pump fluences for 9.2 and $2.5 \mathrm{~nm}$ AMNs. Inset panel (d): Transient absorption data for $2.5 \mathrm{~nm}$ AMNs on a longer time scale. The red and black lines represent, respectively, the instrument response time (pump-probe cross-correlation) and the fits according to the functions described in the Supporting Information.

a

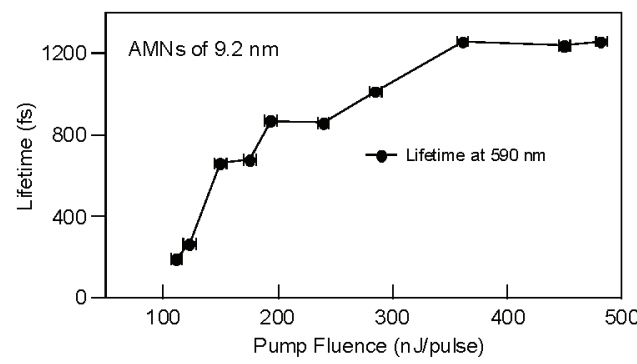

$\mathrm{C}$

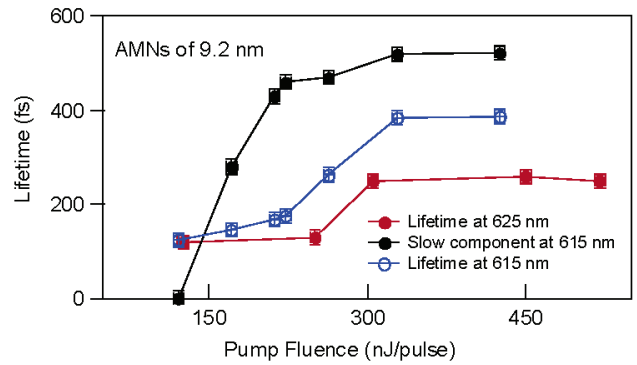

b

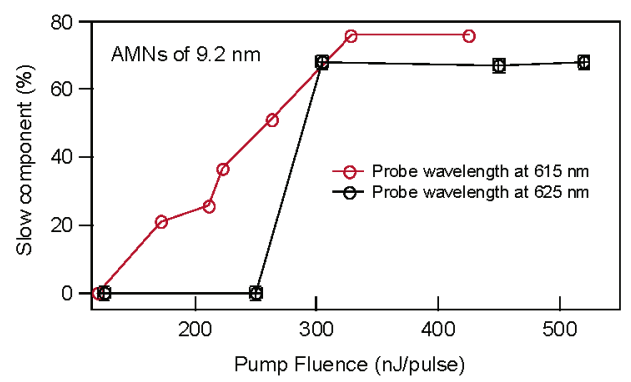

$\mathrm{d}$

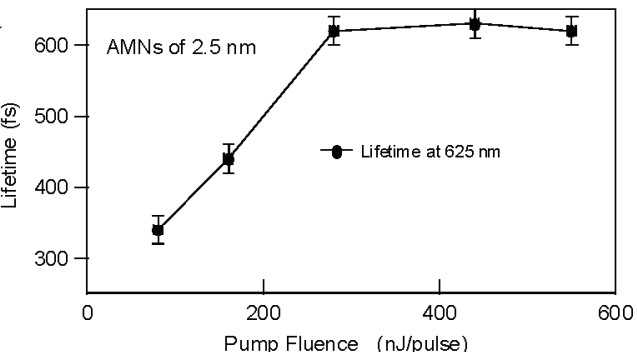

Figure 3. Internal thermalization times and proportions of slow component in the signal rise as a function of pump fluence (see Supporting Information for the extraction procedure of the internal thermalization times).

due to electron-phonon coupling dynamics $(\sim 1 \mathrm{ps})$. Furthermore, an examination of the proportion of the negative part of the signal as a function of the pump fluence indicates that the change of dynamics is not linked to changes in the shape of the spectra. For data obtained at 615 and $625 \mathrm{~nm}$, the proportion of slow components increases with the pump fluences as shown in Figure 3b. For example, for a probe at $615 \mathrm{~nm}$, the internal thermalization time as well as the proportion of the slow component increase from 125 to 385 fs and from $0 \%$ to $75 \%$, respectively, for pump fluences varying from 120 to $425 \mathrm{~nJ} /$ pulse (see Figure 3b,c). A similar behavior is observed with a probe at $625 \mathrm{~nm}$, however, with faster dynamics and a lower proportion of the slow component. Figure $3 \mathrm{~d}$ indicates that the internal thermalization times vary from 340 to $630 \mathrm{fs}$ depending on the pump fluence at $625 \mathrm{~nm}$ for $2.5 \mathrm{~nm}$ AMNs.

From the experimental results presented above, it appears that the dynamics of internal thermalization in these adsorbatecovered metal NPs exhibits anomalous behavior with respect 

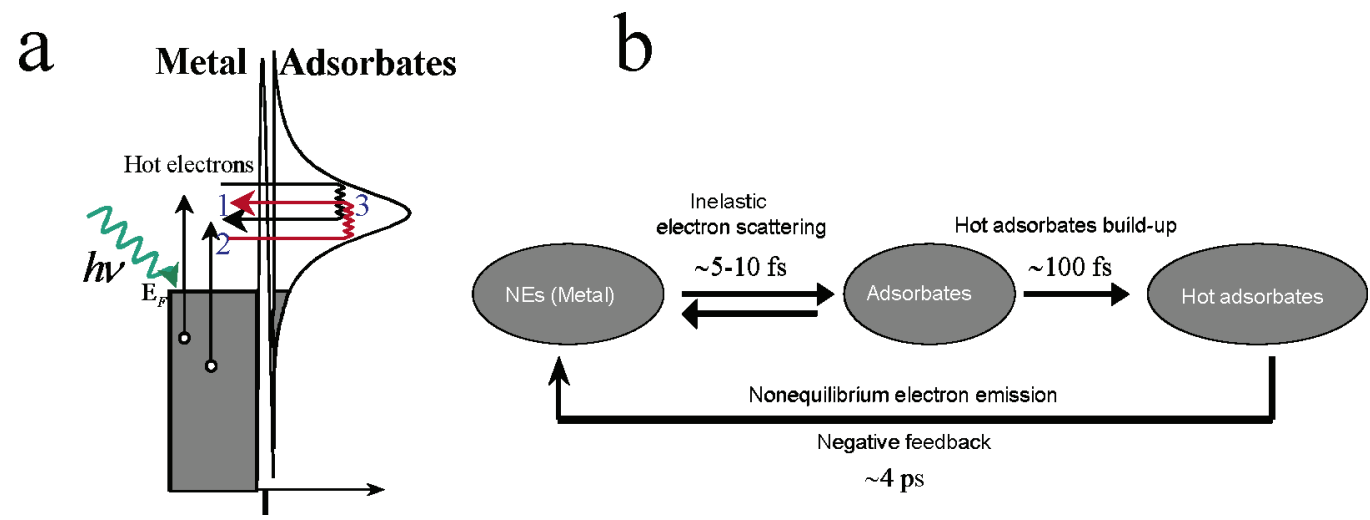

Figure 4. (a) Schematic energetic diagram illustrating the inelastic electron scattering processes between the metal and the adsorbates: (1) inelastic electron scattering into empty electronic states of adsorbates with energy $\left(E_{\mathrm{i}}\right)$; $(3)$ hot electron residence into adsorbates with dissipation (black arrows) or gain of energy (red arrows) $\Delta E=E_{\mathrm{i}}-E_{\mathrm{f}}$; (2) scattering back into the metal conduction band with energy ( $E_{\mathrm{f}}$ ). The black arrows show an incident nonequilibrium electron dissipating energy in the adsorbates ( $\Delta E$ positive), whereas the red arrows illustrate an incident nonequilibrium electron gaining energy ( $\Delta E$ negative) by scattering back from a hot adsorbate. (b) Schematic illustration of the interconnection between the dynamical processes. NEs stands for nonequilibrium electrons (hot electron) and e-MV for electron-molecular vibration interaction.

to bulk gold ${ }^{20,21}$ and naked gold NPs:22,23 (i) At high pump fluence, the internal thermalization dynamics is slower for 2.5 nm AMNs compared to bulk gold; (ii) the internal thermalization time increases with the pump fluence; (iii) two components are present for 9.2 AMNs; (iv) the internal thermalization times are longer for $2.5 \mathrm{~nm}$ compared to $9.2 \mathrm{~nm}$ AMNs; (v) a plateau is present for $2.5 \mathrm{~nm}$ AMNs from $\sim 1.5$ to $\sim 4.5 \mathrm{ps}$.

All these features can be rationalized by the effect of the hot sulfates, acting as a transient energy reservoir that slowly release energy back to the metal electrons. Indeed, the existence of a coupling between the gold electrons and the sulfate vibrations via the partial occupation of the sulfate $\pi^{*}$ orbital differentiates the present system from naked metal NPs. In addition, the increase of the internal thermalization time with the pump fluence suggests that energy of NEs is transiently stored in an energy reservoir, which is coupled to metal electrons. The buildup of a population of hot adsorbates results from the excitation of sulfate vibrations by inelastic nonequilibrium electron scattering into and out of the sulfate $\pi^{*}$ orbital, within the frame of the multiple sequential electronic transition ${ }^{29,30}$ or electronic friction model ${ }^{31-33}$ (see Figure 4a). After the buildup of the population of hot adsorbates, which occurs in hundreds of femtoseconds, ${ }^{30,15,33}$ the subsequent inelastic electron scattering occurs into vibrationally excited sulfates. Thus, NEs can gain kinetic energy when scattering into hot adsorbates by reabsorbing energy deposited earlier during the previous inelastic scattering events. In other words, hot adsorbates can emit nonequilibrium electrons. The time scale of interaction between NEs and adsorbates is on the order of $5-10 \mathrm{fs},{ }^{34,15}$ whereas the lifetime of hot adsorbates on metal surfaces corresponds to a few picoseconds. ${ }^{35-38}$ Thus, the lifetime of the transient reservoir is about 3 orders of magnitude larger than the duration of the system/reservoir interaction. We note that there are two other possible energy reservoirs besides the adsorbates: the "cold" conduction electrons and the lattice. Thus, the whole dynamics corresponds to a competition between electron-molecular vibrations and electron-electron and electron-phonon interactions. As the size of the AMNs decreases, the electron-electron scattering process will be less efficient than electron-MV (molecular vibration) interactions. The presence of both components for the $9.2 \mathrm{~nm}$ AMNs ( 22 000 gold atoms with 13\% of these total gold atoms forming the surface) may illustrate the competition between cold metal electrons (fast component) and the adsorbates (slow component) as a reservoir for the energy dissipation of NEs. The electron-phonon coupling is known to be very weak within the nonthermal regime ${ }^{39}$ and does not play a significant role on the time scale of interest here.

The proposed mechanism is supported by recent reports demonstrating that highly vibrationally excited adsorbates can emit nonequilibrium electrons in a metal by a coupling involving a charge transfer. ${ }^{40-42}$ In the present AMNs, the energy stored in the hot adsorbates is also converted into hot electrons in gold by a coupling mechanism involving the partial occupation of the sulfate $\pi^{*}$ orbital by gold electrons. Finally, the hot adsorbates operate a negative feedback, since they respond to reverse the direction of the change (energy dissipation of NEs) by transferring energy back to NEs, leading to an inhibition of the internal thermalization. Our results indicate that the negative feedback can be enhanced by increasing the pump fluence within regime $\mathrm{A}$ or by decreasing the size of $\mathrm{AMNs}$ within regime $\mathrm{B}$. In regime $A$, as the pump fluence is increased, the effect of energy reabsorption by NES from the hot sulfates becomes more important and thus changes the dynamics. Within regime B, the dynamics essentially reflects the hot adsorbates relaxation dynamics leading to a pump fluence independent behavior. Indeed, we note that the dynamical locking time is comparable to the time scale of a vibrationally excited adsorbates molecule on metal surfaces (typically a few picoseconds). ${ }^{36}$ A similar behavior is observed in semiconductors where the lifetime of the hot phonons, acting as a transient energy reservoir, controls the hot charge carrier cooling dynamics. ${ }^{10,43}$ Evidence for the control of NE dynamics in the metal by the transient energy reservoir (hot adsorbates) comes from the data obtained with 2.5 AMNs shown in the inset of Figure $2 \mathrm{~d}$. As indicated by the similar rise time and the plateau, the dynamics are locked during about 4.5 picoseconds, then a change between the two signals during the decay can be observed. This effect is understood as follows: Since the dynamics of electron-phonon coupling is pump fluence dependent, a difference can be observed when the dynamical locking by the hot adsorbates is no longer operating. The duration of dynamical control is given by the lifetime of the vibrational adsorbates, which is governed by the metal phonon-vibrational modes coupling, the heat dissipation towards the surrounding medium, and the substrate electronic excitation. Figures 4 summarizes the mechanism involved in the retardation of the internal thermalization based on the reabsorption effect by the metal electrons of the energy stored in the hot sulfates, which have a much longer lifetime compared to NEs (see Figure 4a). As long as the adsorbates are in a 
vibrationally excited state (during $\sim 4 \mathrm{ps}$ ), they can emit nonequilibrium electrons ${ }^{41}$ leading to the negative feedback loop (see Figure 4b). A strong manifestation of the negative feedback is revealed by the plateau observed between 1.5 and $4.5 \mathrm{ps}$ for the $2.5 \mathrm{~nm}$ AMNs, illustrating the stabilization of the electronic temperature (still higher than the lattice temperature) during the lifetime of the hot adsorbates. Indeed, the purpose of a negative feedback loop in a system is to stabilize the system against external stimuli. ${ }^{44}$

Acknowledgment. The authors are grateful to P. Comte for the gift of metal oxide films; V. Thorsmolle, B. Wenger, J.-E. Moser, A. Aebischer, A. Agrios, and N. Eugster for helpful comments; and V. Devaud for technical assistance. The Swiss National Research Foundation and the State Secretariat for Education and Research are acknowledged for financial support. This work is part of the TMR network SUSANA (Supramolecular Self-Assembly of Interfacial Nanostructures).

Supporting Information Available: Sample preparation method, SEM and TEM images, and FT-IR spectra. Discussion on the optical response of nonequilibrium electrons and the fitting procedure for the extraction of internal thermalization times. This material is available free of charge via the Internet at http://pubs.acs.org.

\section{References and Notes}

(1) Heinglein, A. J. Phys. Chem. 1993, 97, 5457-5471.

(2) Zhang, P.; Sham, T. K. Appl. Phys. Lett. 2002, 81, 736-738.

(3) Bosbach, J.; Hendrich, C.; Stietz, F.; Vartayan, T.; Träger, F. Phys. Rev. Lett. 2002, 89, 257404.

(4) Zhang, P.; Sham, T. K. Phys. Rev. Lett. 2003, 90, 245502.

(5) Hövel, H.; Fritz, S.; Hilger, A.; Kreibig, U. Phys. Rev. B 1993, 48, 18178

(6) Persson, B. N. J. Surf. Sci. 1993, 283, 153.

(7) Kreibig, U.; Vollmer, M. Optical Properties of Metal Clusters; Springer-Verlag: Berlin, 1995. 264.

(8) Patrito, E. M.; Paredes, O., P.; Sellers, H. Surf. Sci. 1997, 380,

(9) Bauer, C.; Abid, J.-P.; Fermin, D.; Girault, H. H. J. Chem. Phys. 2004, $120,9302$.

(10) Shah, J. Ultrafast Spectroscopy of Semiconductors and Semiconductors Nanostructures; Springer-Verlag: Berlin, 1999; p 172.

(11) Bonn, M.; Funk, S.; Hess, C.; Denzler, D. N.; Stampf, C.; Scheffler, M.; Wolf, M.; Ertl, G. Science 1999, 285, 1042.

(12) Joachim, C.; Gimzewski, J. K.; Aviram, A. Nature (London) 2000 $408,541$.

(13) Nitzan, A.; Ratner, M. A. Science 2003, 300, 1384.

1402 .
(15) Petek, H.; Nagano, H.; Weida, M. J.; Ogawa, S. J. Phys. Chem. B 2001, 105, 6767.

(16) Bartels, L.; Wang, F.; Möller, D.; Knoessel, E.; Heinz, T. F. Science 2004, 305, 648

(17) Logunov, S. L.; Ahmadi, T. S.; El-Sayed, M. A.; Khoury, J. T.; Whetten, R. L. J. Phys. Chem. B 1997, 101, 3713-3719.

(18) Hartland, G. V.; Hu, M.; Sader, J. E. J. Phys. Chem. B 2003, 107, $7472-7478$.

(19) Fann, W. S.; Storz, R.; Tom, H. W. K.; Bokor, J. Phys. Rev. B 1992, 46, 13592.

(20) Sun, C. K.; Vallée, F.; Acioli, L. H.; Ippen, E. P.; Fujimoto, J. G. Phys. Rev. B 1994, 50, 15337.

(21) Del Fatti, N.; Voisin, C.; Achermann, M.; Tzortakis, S.; Christofilos, D.; Vallée, F. Phys. Rev. B 2000, 61, 16956.

(22) Voisin, C.; Christofilos, D.; Fatti, N. D.; Vallee, F.; Prevel, B.; Cottancin, E.; Lerme, J.; Pellarin, M.; Broyer, M. Phys. Rev. Lett. 2000 $85,2200-2203$.

(23) Voisin, C.; Christofilos, D.; Loukakos, P. A.; Del Fatti, N. D.; Vallée, F.; Lermé, J.; Gaudry, M.; Cottancin, E.; Pellarin, M.; Broyer, M. Phys. Rev. B 2004, 69, 195416.

(24) Rolison, D. R. Science 2003, 299, 1698 545.

(26) Quinn, J. J. Phys. Rev. 1962, 126, 1453.

(27) Rosei, R.; Antonangeli, F.; Grassano, U. M. Surf. Sci. 1973, 37, 689.

(28) Dobryakov, A. L.; Farztdinov, V. M.; Lozovik, Y. E.; Marowski, G. Phys. Scr. 1999, 60, 572 .

(29) Misewich, J. A.; Heinz, T. F.; Newns, D. M. Phys. Rev. Lett. 1992 68, 3737.

(30) Prybyla, J. A.; Tom, H. W. K.; Aumillar, G. D. Phys. Rev. Lett. 1992, 68, 503.

(31) Gadzuk, J. W. Chem. Phys. 2000, 251, 87.

(32) Brandbyge, M.; Hedegard, P.; Heinz, T. F.; Misewich, J. A.; Newns, D. M. Phys. Rev. B 1995, 52, 6042.

(33) Tully, J. C. Аnnu. Rev. Phys. Chem. 2000, 51, 153

(34) Gadzuk, J. W. Surf. Sci. 1995, 342, 345.

(35) Harris, A. L.; Rothberg, L.; Dubois, L. H.; Levinos, N. J.; Dhar, L. Phys. Rev. Lett. 1990, 64, 2086.

(36) Cavanagh, R. R.; King, D. S.; Stephenson, J. C.; Heinz, T. F. J. Phys. Chem. B 1993, 97, 786.

(37) Cavanagh, R. R.; Heiweil, E. J.; Stephenson, J. C. Surf. Sci. 1994, 299/300, 643

(38) Germer, T. A.; Stephenson, J. C.; Heilweil, E. J.; Cavanagh, R. R. J. Chem. Phys. 1994, 101, 1704

(39) Groeneveld, R. H. M.; Sprik, R.; Lagendijk, A. Phys. Rev. B 1992, $45,5079$.

(40) Gergen, B.; Nienhaus, H.; Weinberg, W. H.; McFarland, E. W. Science 2001, 294, 2521.

(41) White, J. D.; Chen, J.; Matsiev, D.; Auerbach, D. J.; Wodtke, A. M. Nature (London) 2005, 433, 503.

(42) Nienhaus, H. Surf. Sci. Rep. 2002, 45, 3-78.

(43) Langot, P.; Del Fatti, N.; Christofilos, D.; Tommasi, R.; Vallée, F. Phys. Rev. B 1996, 54, 14487.

(44) Bechhoefer, J. Rev. Mod. Phys. 2005, 77, 783-836 and refs. therein. 